\title{
Optimal Design of an IPMSM for High-Speed Operation Using Electromagnetic and Stress Analysis
}

\author{
Jang-Ho Seo ${ }^{\dagger}$ and Hyun-Kyo Jung*
}

\begin{abstract}
In the development of an interior permanent magnet synchronous machine (IPMSM) for high-speed operation, the problem of mechanical stress of the rotor by centrifugal force becomes more essential as the speed and size of the machines increase. In this paper, the optimal design process combined with mechanical stress analysis was presented. In the analysis of mechanical stress, the node and element data obtained by the electromagnetic field analysis program are also used in the stress analysis. Therefore, the different pre-processing for the stress analysis program is no longer required. Therefore, the computing time of the new method is very short compared with the conventional approach, and when repeated analyzes of various models are required, this method is very useful. The validity of our methods was verified by comparing simulation results with conventional and experimental data.
\end{abstract}

Keywords: Centrifugal force, finite element analysis, mechanical stress, interior permanent magnet synchronous machine

\section{Introduction}

With developments in power electronics and improvements in permanent magnet performance, the interior permanent magnet synchronous machine (IPMSM) using a field-weakening control has been a good candidate for high-speed applications [1-3]. The IPMSM, which has its magnet located inside the rotor core, has many advantages, such as high efficiency, high power density, high torque density and a wide speed range, among others.

Meanwhile, in the conventional design of the IPMSM, the electromagnetic field analysis is generally separated from the mechanical stress analysis. Furthermore, in the optimization process, it is very difficult to integrate each analysis into one process.

In our previous research, the stress calculation was carried out by the summation of each centrifugal force which each element receives at the whole specific region predetermined by designer [3]. This is very useful in evaluating stress in the rotor core. However, it cannot consider distribution of displacements and distribution of stress in all regions. From this point of view, we propose the twodimensional numerical stress analysis which can solve the drawbacks of the previous method.

In the newly proposed method, the different preprocessing for each analysis program is no longer required by combining field analysis programs in parallel. In other words, the node and element data obtained by the electromagnetic field analysis program is also used in the stress analysis.

$\dagger$ Corresponding Author : School of Electrical Engineering and Computer Science, Seoul National University, Korea.

(jangho78@elecmech.snu.ac.kr)

* School of Electrical Engineering and Computer Science, Seoul National University, Korea. (hkjung @snu.ac.kr)

Received 7 January 2009; Accepted 4 July 2009
Therefore, the computing time of the new method is very short in comparison with that of the conventional program while the results of the proposed method are similar to those of the conventional method. In particular, when the repeated analyzes of various models are required, this method is very useful and convenient.

\section{Analysis Method}

\subsection{Analysis Model of Electric Machine}

Fig. 1 shows an analysis model with one magnet layer in the rotor core. Generally, the shape of the layer and length are designed to enhance the capability of the IPMSM such as with high reluctance torque and sinusoidal EMF, etc.

In high-speed operations, the center-post and bridge sustaining the magnet have the potential to be broken by the stress caused by centrifugal force. Therefore, the length of the bridge and center-post should have sufficient thickness to prevent the dispersion of the magnets.

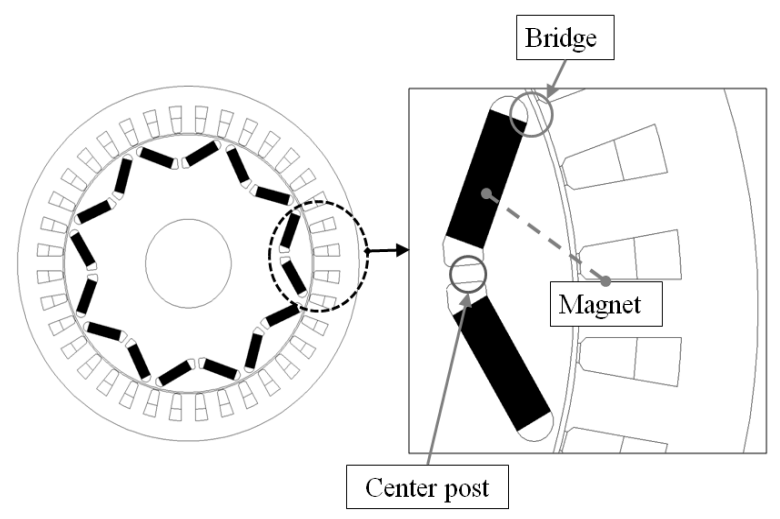

Fig. 1. Configuration of IPMSM. 


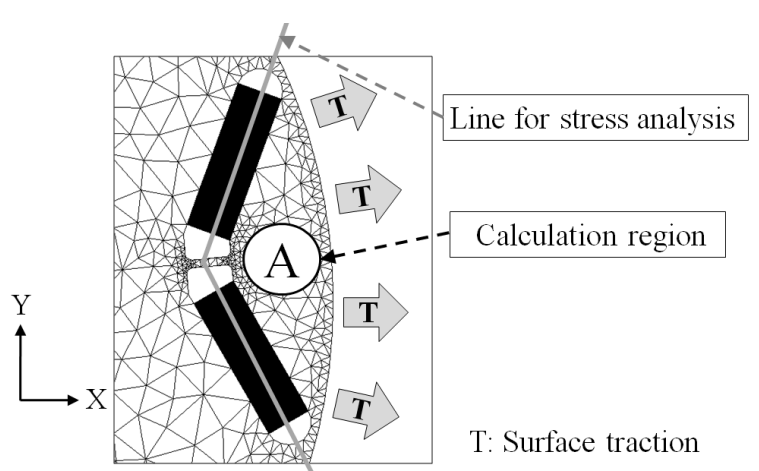

Fig. 2. "A" region for stress analysis. The line regulates the particular "A" region to be analyzed for centrifugal force.

\subsection{Previous Research}

In our previous research, the stress calculation of the IPMSM was carried out by the summation of each force of an element in "A" region. The specific "A" region for calculating total stresses was predetermined by the designer as shown in Fig. 2. The node and element information corresponding to "A" region were obtained respectively from the magnetic field analysis program. The formula of the centrifugal force for previous stress analysis can be expressed as follow [3]:

$$
F=\sum_{n=1}^{N}\left(\rho \times A_{n} \times l \times r_{n} \times \omega^{2}\right)
$$

where, $N$ is the total number of elements corresponding to " $\mathrm{A}$ " region, $\rho$ is the material density, $A_{n}$ is the element area of $n$ th, $l$ is the stack length, $r_{n}$ is the distance from the origin, and $\omega$ is the angular velocity.

Centrifugal force was used to evaluate average mechanical stress by dividing the area of the center-post and bridge. When the optimization for design of the IPMSM is required, this method is effective due to its easy realization of stress analysis.

However, this strategy had a potential problem in that it is difficult to estimate the area of the center-post and bridge accurately. In addition, it cannot consider the distribution of displacements and stress in all regions.

\subsection{Stress Analysis Method based on FEA}

The IPMSM faces the mechanical stress caused by centrifugal force. When the rotor speed is very high, the force can deform the rotor core or break the center-post and bridge.

Therefore, it is important to consider the distribution of the stress and the displacements for the machine designer before manufacturing the IPMSM.

In the stress analysis, we assumed our problem can be considered as plane stress. Plane stress is defined to be a state of stress in which the normal stress and the shear stress directed perpendicular to the plane are assumed to be zero. For instance, in Fig. 2, the rotor plate in the x-y plane shown subjected to surface traction $\mathrm{T}$ in the plane is under a state of plane stress: that is, the normal stress $\sigma_{z}$ and the shear stresses $\tau_{x z}$ and $\tau_{y z}$ are assumed to be zero. Generally, problems whose loads act only in the x-y plane can be considered to be under plane stress.

The flow of the stress analysis is as follows:

\section{Stage 1: Select Displacement Functions.}

The element displacement functions are taken to be

$$
\begin{aligned}
& u(x, y)=a_{1}+a_{2} x+a_{3} y \\
& v(x, y)=a_{4}+a_{5} x+a_{6} y
\end{aligned}
$$

The linear function ensures that the displacements along the edge and at the nodes shared by adjacent elements are equal. Meanwhile, the nodal displacement matrix is given by

$$
\{d\}=\left\{\begin{array}{l}
d_{i} \\
d_{j} \\
d_{m}
\end{array}\right\}=\left\{\begin{array}{l}
u_{i} \\
v_{i} \\
u_{j} \\
v_{j} \\
u_{m} \\
v_{m}
\end{array}\right\}
$$

The general displacement function $\{\psi\}$, which stores the functions $u$ and $w$, can be expressed as

$$
\{\psi\}=\left\{\begin{array}{l}
u(x, y) \\
v(x, y)
\end{array}\right\}=\left\{\begin{array}{lll}
N_{i} u_{i} & N_{j} u_{i} & N_{m} u_{m} \\
N_{i} v_{i} & N_{j} v_{i} & N_{m} v_{m}
\end{array}\right\}
$$

where, $N_{i}, N_{j}$, and $N_{m}$ are linear shape functions. Finally, expressing (3) in abbreviated matrix form, we have

$$
\{\psi\}=[N]\{d\}
$$

Stage 2: Define the strain/displacement and Stress/Strain Relationship.

The strain associated with the two-dimensional element is given by

$$
\begin{aligned}
\{\varepsilon\} & =\left\{\begin{array}{l}
\varepsilon_{x} \\
\varepsilon_{y} \\
\gamma_{x y}
\end{array}\right\}=\left\{\begin{array}{c}
\frac{\partial u}{\partial x} \\
\frac{\partial v}{\partial y} \\
\frac{\partial u}{\partial y}+\frac{\partial v}{\partial x}
\end{array}\right\}=\frac{1}{2 A}\left[\begin{array}{cccccc}
\beta_{i} & 0 & \beta_{j} & 0 & \beta_{m} & 0 \\
0 & \gamma_{i} & 0 & \gamma_{j} & 0 & \gamma_{m} \\
\gamma_{i} & \beta_{i} & \gamma_{j} & \beta_{j} & \gamma_{m} & \beta_{m}
\end{array}\right]\left[\begin{array}{c}
u_{i} \\
v_{i} \\
u_{j} \\
v_{j} \\
u_{m} \\
v_{m}
\end{array}\right] \\
& =[B]\{d\}
\end{aligned}
$$

where $\varepsilon_{x}$ is the normal strain in the x axes direction, and $\varepsilon_{y}$ is the normal strain in the y axes direction $\gamma_{x y}$ is the shear strain in the $x-y$ plane. The stress/strain relationship is given by

$$
\{\sigma\}=\frac{E}{1-v^{2}}\left[\begin{array}{ccc}
1 & v & 0 \\
v & 1 & 0 \\
0 & 0 & \frac{1-v}{2}
\end{array}\right]\{\varepsilon\}=[D]\{\varepsilon\}
$$
ratio.

where $E$ is the modulus of elasticity, and $v$ is poisson`s

Using (5) and (6), we obtain the stresses in terms of the unknown nodal degrees of freedom as

$$
\{\sigma\}=[D \rrbracket B]\{d\}
$$



tions.

Stage 3: Derive the Element Stiffness Matrix and Equa-

Using the principle of minimum potential energy, the element stiffness matrix can be derived as follows:

$$
[k]=\iiint_{V}[B]^{T}[D][B] d V
$$

Stage 4: Assemble the Element Equations to Obtain the Global Equations.

We obtain the global structure stiffness matrix and equation by using the direct stiffness method as

$$
\begin{gathered}
{[K]=\sum_{e=1}^{N}\left[k^{(e)}\right]} \\
\{F\}=\sum_{e=1}^{N}\left[f^{(e)}\right]
\end{gathered}
$$

$\{F\}$ is the column of equivalent global nodal loads obtained by lumping body forces $f^{(e)}$.

The body force at the nodes can be evaluated as

$$
\begin{gathered}
\left\{f^{(e)}\right\}=\iiint_{V}[N]^{T}\{X\} d V \\
\{X\}=\left\{\begin{array}{c}
X_{b} \\
Y_{b}
\end{array}\right\} \\
X_{b}=\rho \omega^{2} x_{e}, Y_{b}=\rho \omega^{2} y_{e}
\end{gathered}
$$

Where $X_{b}$ and $Y_{b}$ are the weight densities in the $\mathrm{x}$ and $\mathrm{y}$ directions, respectively, and $\rho$ is mass density, and $\omega$ is angular velocity, and $x_{e}$ and $y_{e}$ are the center of element in the $\mathrm{x}$ and $\mathrm{y}$ directions, respectively.

\subsection{Comparison of Measured and Calculated Value}

To verify the stress analysis method, an IPMSM with a 4-pole prototype machine having four layers was selected. The specification of the prototype is shown in Table 1. Fig. 3 shows the structure of the IPMSM and its experimental devices.

In the rotor structure, permanent magnets were partially inserted into each layer to obtain the sinusoidal back-EMF waveform. The shape and length of each layer was designed to enhance the inductance difference. The material which was used is nonoriented silicon steel (S18) suitable for a rotating electrical machine. Its yield strength is 300 [MPa].

The IPMSM was operated at 18000 [rpm] with intent to break the rotor core structure in order to study the effect of mechanical stress. The broken model and analysis results at high-speed test are shown in Fig. 4. The comparison of computational results is summarized in Table 2.

As a simulation result, the maximum stress calculated by the stress analysis was $519[\mathrm{MPa}]$ at the center-post. This value is larger than the yield strength and tensile strength of the steel. Also, the location with maximum value coincides with the broken region. However, the maximum value estimated by the conventional method is smaller than the yield strength. Therefore, it can't explain the reason for the broken region.

From the simulation results, we can see that our methods agree well with the experimental ones compared to the conventional method.

Table 1. Specifications of prototype model

\begin{tabular}{cc}
\hline Parameters and Constraints & Value \\
\hline Number of poles & 4 \\
Number of slots & 24 \\
Rated power & $6[\mathrm{~kW}]$ \\
Maximum speed & $18000[\mathrm{rpm}]$ \\
Used silicon steel & $\mathrm{S} 18$ \\
\hline
\end{tabular}

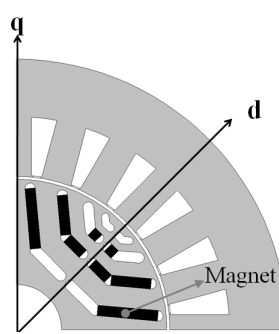

(a) Cross section of IPMSM

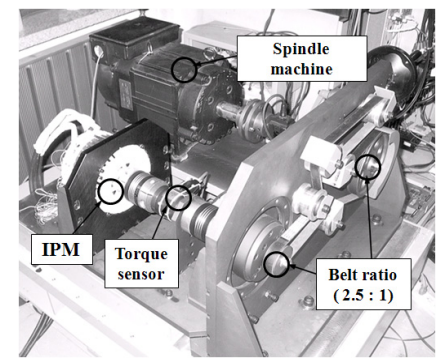

(b) Experimental setup
Fig. 3. Configuration of IPMSM with experimental devices.

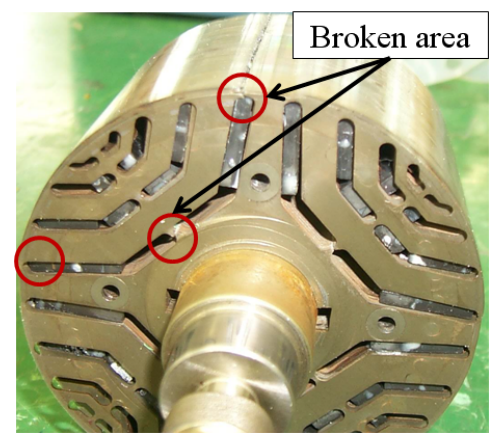

(a) Broken model

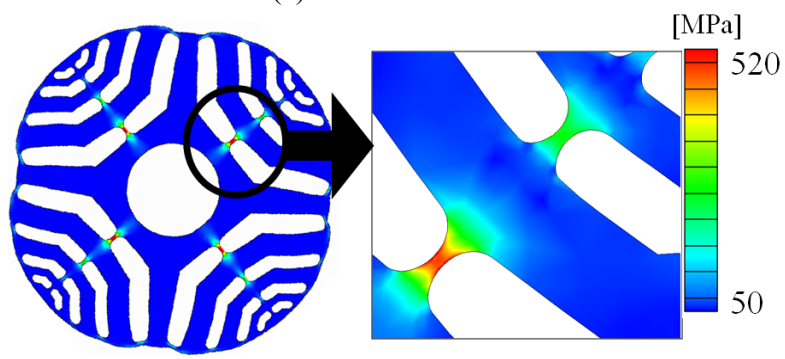

(b) Stress analysis result

Fig. 4. Broken model and analysis results at high-speed test (18000[rpm]).

Table 2. Computational analysis results

FEA

Conventional

Maximum stress [MPa]

519

285

Maximum displacement

0.02 [mm] 


\section{Optimal Design and Result}

\subsection{Objective Function and Design variables}

As a practical optimization example, an IPMSM for an electric vehicle was selected. The initial structure of the analysis model is presented in Fig. 5. The objective of the optimal design to be maximized was defined by

$$
\text { Torque }=\frac{3}{2} \frac{P}{2}\left\{\left(L_{d}-L_{q}\right) i_{d} i_{q}+\lambda_{f} i_{q}\right\}[N . m]
$$

where $L_{d}$ and $L_{q}$ are d- and q- axis inductances. $i_{d}$ and $i_{q}$ are $\mathrm{d}$ - and q-axis currents. $\lambda_{f}$ is magnet flux linkage.

If the value of machine torque at starting is similar, a solution with a lower total harmonic distortion (THD) of EMF, lower amplitude of EMF and lower iron loss is preferred. The torque can be evaluated by (13) and thus easily applied to the optimization process; whereas the THD of EMF, peak of EMF, iron loss require additional numerical computations and thus is hard to apply to the optimization process. Therefore, in this study, we first selected several solutions which have high machine torque, and then calculated the THD of EMF, peak of EMF, and iron loss only for the candidate solutions.

To protect inverter devices in case of a fault condition at maximum speed, the magnet quantity should be constrained. When the machine is operated at high-speed, its efficiency is reduced and magnet dispersion may occur. Therefore, the iron loss and maximum stress of the machine also should be constrained. The stress constrain used in the optimization process was yield strength of the steel at $12000[\mathrm{rpm}]$.

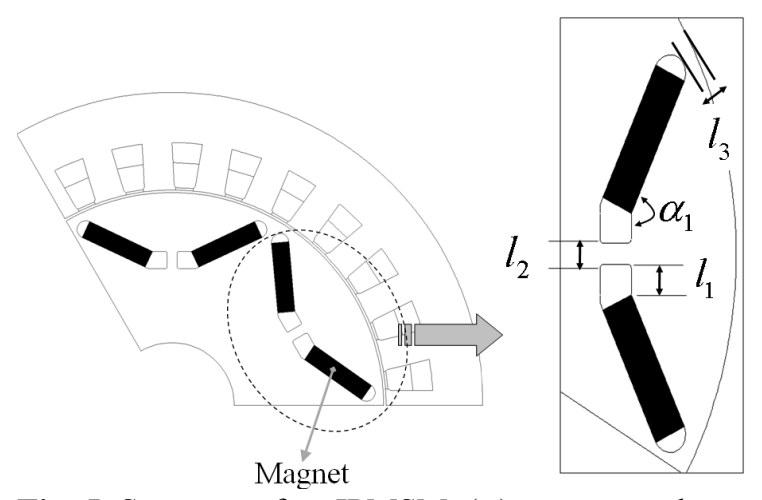

Fig. 5. Structure of an IPMSM, $(\alpha)$ : magnet pole arc angle, $\left(l_{1}, l_{2}, l_{3}\right)$ : layer length, center-post length, bridge length).

Table 3. Specifications of objective machine

\begin{tabular}{cc}
\hline Rated power & $100[\mathrm{~kW}]$ \\
Rated RPM & $12000[\mathrm{rpm}]$ \\
Stator outer radius & $250[\mathrm{~mm}]$ \\
Stacking length & $230[\mathrm{~mm}]$ \\
Air gap length & $1[\mathrm{~mm}]$ \\
Permanent magnet & Sintered NdFeB, $1.2[\mathrm{~T}]$ \\
\hline
\end{tabular}

Due to the limit condition, we selected 4 parameters concerning with reluctance torque, which has an important role in increasing the starting torque.

Magnet pole arc angle $(\alpha)$, layer length $\left(l_{l}\right)$, center-post length $\left(l_{2}\right)$, and bridge length $\left(l_{3}\right)$ were taken as design variables. The design variables are shown in Fig. 5. Table 3 shows the specification of the objective machine.

\subsection{Optimization Process}

The magnetic field analysis and the stress analysis are based on 2-D finite element analysis. The node and element data obtained by the electromagnetic field analysis program is also used in the stress analysis. Therefore, the different pre-processing for the stress analysis program is no longer required because these analyses use the same nodes and elements.

In this research, multi-grouped particle swarm optimization (MGPSO) was used for the optimal design [1].

Fig. 6 shows the whole optimization process. If design variables are given from MGPSO, 2-D magnetic field analysis with FEM is performed from which objective function and constraints are calculated.

Meanwhile, using the FEM information by the pre and post process of the magnetic field analysis, the stress analysis is performed at once. The optimization process stopped when the number of generations decreased below the convergence condition.

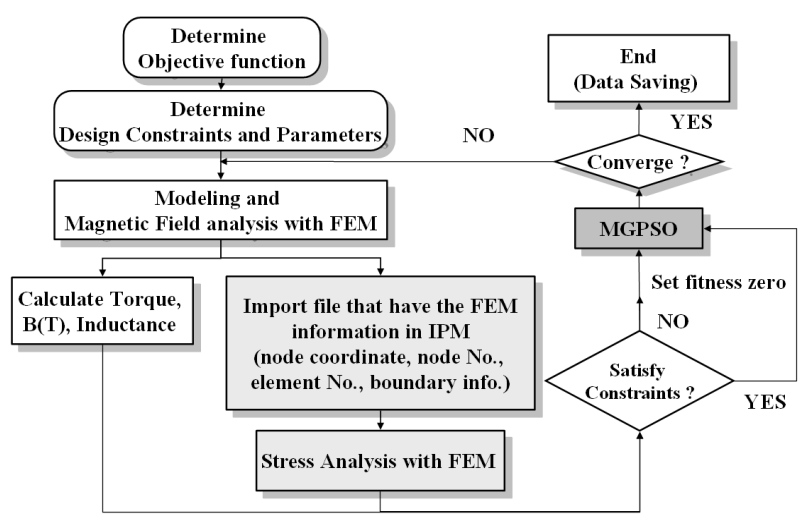

Fig. 6. Total IPMSM optimization process.

Table 4. Optimization results

\begin{tabular}{cccc}
\hline Solutions & Case 1 & Case 2 & Case 3 \\
Starting torque $[\mathrm{Nm}]$ & 382 & 372 & 379 \\
THD of EMF [\%] & 5.2 & 4.6 & 5.5 \\
EMF [V] & 660.0 & 702.2 & 685.3 \\
Iron loss [W] & 850 & 905 & 833 \\
$l_{1}[\mathrm{~mm}]$ & 2.2 & 5 & 1.5 \\
$l_{2}[\mathrm{~mm}]$ & 1.0 & 1.6 & 1.7 \\
$l_{3}[\mathrm{~mm}]$ & 1.6 & 1.5 & 1.1 \\
$\alpha_{1}\left[^{\circ}\right]$ & 155.5 & 149.5 & 152.0 \\
\hline
\end{tabular}

\subsection{Optimization Results}

Table 4 shows the three superior solutions optimized for maximum machine torque. Then, the THD of EMF, peak 
of EMF, and iron loss were evaluated for each candidate solution. From the table, we can see that case 1 is the best compromise solution because it has a sufficiently high starting torque, which is as comparable as the other solutions, small EMF, low THD of EMF, and low iron loss.

\section{Conclusion}

In this research, the optimal design process of the IPMSM combined with mechanical stress analysis was presented. Through the proposed stress analysis method using electromagnetic finite element analysis information, it is possible to integrate each analysis into one optimization program in parallel as subroutine and to predict mechanical stability in the primary design stage.

\section{Acknowledgements}

This research was supported by a grant (Code07 Next Generation High Speed Train A01) from the Railroad Technology Development Program (RTDP) funded by the Ministry of Land, Transport and Maritime Affairs of the Korean government.

\section{References}

[1] J. H. Seo, C. H. Im, C. G. Heo, J. K. Kim, H. K. Jung, and C. G. Lee, "Multimodal function optimization based on particle swarm optimization", IEEE Tans. Magn, Vol. 42, No. 4, pp. 1095-1098, 2006.

[2] G. X. Zhou, R.Y. Tang, D. H. Lee, and J. W. Ahn, "Field circuit coupling optimization design of the main electromagnetic parameters of permanent magnet synchronous motor", JEET, Vol. 3, No. 1, pp. 8893, 2008.

[3] S. M. Cho, J. K. Kim, H. K. Jung, and C. G. Lee, "Stress and thermal analysis coupled with field analysis of multilayer buried magnet synchronous machine with a wide speed range", IEEE Tans. Magn., Vol. 41, No. 5, pp. 1632-1635, May 2005.

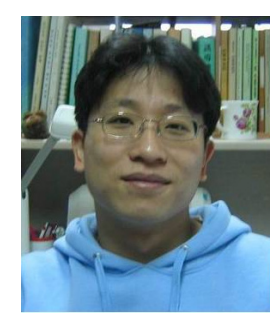

Jang-Ho Seo received his B.S. degree in electrical engineering from ChoongAng University. His research interests are numerical analysis and design of electrical machines.

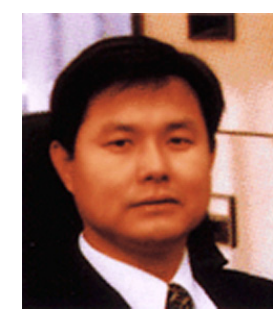

Hyun-Kyo Jung received his Ph.D. degree in electrical engineering from Seoul National University. His research interests are electric machinery, EM waves, circuit breakers, and so on. 